Introduction and Objective Aclidinium bromide is a novel, longacting, muscarinic antagonist indicated as a maintenance treatment for chronic obstructive pulmonary disease (COPD). Maintaining significant bronchodilation throughout the 24-hour day is important to improve outcomes for patients with COPD. This study evaluated daily bronchodilatory symptom control with twice-daily (BID) aclidinium in patients with stable, moderate-to-severe COPD. Methods In this 6-week, randomised, double-blind, Phase IIIb study, patients received aclidinium $400 \mu \mathrm{g}$ (metered dose; equivalent to aclidinium $322 \mu \mathrm{g}$ delivered dose) BID, tiotropium bromide $18 \mu \mathrm{g}$ once daily or placebo. Change from baseline in normalised forced expiratory volume in 1 second $\left(\mathrm{FEV}_{1}\right)$ area under the curve over the 24-hour period immediately following morning treatment $\left(\mathrm{AUC}_{0-24}\right)$ at Week 6 was the primary endpoint. Other endpoints included change from baseline in normalised $\mathrm{FEV}_{1} \mathrm{AUC}_{12-24}$ and $\mathrm{AUC}_{0-12}$, predose (trough) $\mathrm{FEV}_{1}$ and peak $\mathrm{FEV}_{1}$. Daily symptoms were recorded each evening using the 11-item EXAcerbations of Chronic pulmonary disease Tool-Respiratory Symptoms (EXACT-RS) and a total score was calculated (range 0-40: more severe symptoms indicated by higher score). Additional daily symptoms, including presence of morning symptoms and night-time symptom severity (5-point scale: $0=$ none; $4=$ very severe), were recorded using electronic diaries.

Results In total, 414 patients were randomised: mean age was $62.3 \pm 8.1$ years (mean $\pm \mathrm{SD}$ ); $54.1 \%$ were current smokers; baseline $\mathrm{FEV}_{1}$ was 1.484 $\pm 0.51 \mathrm{~L}$ At Week 6 , aclidinium $400 \mu \mathrm{g}$ BID and tiotropium $18 \mu \mathrm{g}$ OD significantly improved lung function from baseline compared with placebo (Table). Compared with placebo, aclidinium and tiotropium significantly reduced EXACT-RS total scores from baseline $(-2.1$ and -1.3 versus $-0.1 ; p<0.0001$ and $p<0.05$, respectively), and increased percentage of days without morning symptoms from baseline $(6.7 \%$ and $3.4 \%$, respectively, versus $-2.2 \%$; $\mathrm{p}<0.05$ for both) at Week 6 . Night-time symptom severity scores were significantly reduced from baseline with aclidinium $(-0.16)$ versus placebo $(-0.02)$ at Week $6(\mathrm{p}<0.05)$ but improvements with tiotropium (-0.09) did not reach statistical significance.

Conclusions Aclidinium $400 \mu \mathrm{g}$ BID provided significant 24-hour bronchodilation and daily symptom improvement throughout the study. The bronchodilatory effect of aclidinium was similar to tiotropium, with numerically greater improvement in morning and night-time symptoms among aclidinium-treated patients.

Abstract S51 Table 1 Spirometric variables at Week 6

\begin{tabular}{lccc}
\hline $\begin{array}{l}\text { Change from baseline vs } \\
\text { placebo, } \mathbf{~ m L}\end{array}$ & $\begin{array}{l}\text { Aclidinium } \\
\mathbf{4 0 0} \boldsymbol{\mu} \mathbf{g}\end{array}$ & $\begin{array}{l}\text { Tiotropium } \\
\mathbf{1 8} \boldsymbol{\mu} \mathbf{g}\end{array}$ & Difference \\
\hline Normalized FEV ${ }_{1} \mathrm{AUC}_{0-24}$ & $150^{\dagger}$ & $140^{\dagger}$ & 10 \\
Normalized FEV $\mathrm{AUC}_{12-24}$ & $160^{\dagger}$ & $123^{\dagger}$ & 37 \\
Normalized FEV $\mathrm{AUC}_{0-12}$ & $138^{\dagger}$ & $156^{\dagger}$ & -18 \\
Morning pre-dose (trough) FEV & $141^{\dagger}$ & $102^{\dagger}$ & 39 \\
Peak FEV & $180^{\dagger}$ & $172^{\dagger}$ & 8 \\
\hline
\end{tabular}

${ }^{*} p<0.05 ;{ }^{\dagger} p<0.0001$.

\section{S52 CLINICAL EFFECTIVENESS OF TELEMONITORING FOR CHRONIC OBSTRUCTIVE PULMONARY DISEASE (COPD): RANDOMISED CONTROLLED TRIAL}

doi:10.1136/thoraxjnl-2012-202678.058

'H Pinnock, 'L McCloughlan, ' ${ }^{1}$ A Todd, ${ }^{2} \mathrm{~J}$ Hanley, 'S Lewis, 'A Krishan, 'W MacNee, ${ }^{1} \mathrm{C}$ Pagliari, 'A Sheikh, 'B McKinstry. 'The University of Edinburgh, Edinburgh, UK; ${ }^{2}$ Edinburgh Napier University, Edinburgh, UK

Introduction Previous trials of telehealthcare for COPD have included enhanced clinical care compared with controls. It is therefore unclear if telehealthcare alone improves clinical outcomes and reduces hospital admissions.
Aim To determine if telemetrically supported self-monitoring of COPD postpones hospital admissions when both intervention and control groups receive optimised care.

Trial design 1-year, researcher-blind RCT in UK primary care. Methods Patients with a COPD admission in the previous year were randomised centrally to telemetric or traditional modes of monitoring: both groups received the same clinical care. The primary outcome, assessed by a researcher blinded to allocation, was time to first hospital admission caused by a COPD exacerbation over the trial year. Other outcomes included number of admissions, bed days, deaths and health-related quality of life (St George's Respiratory Questionnaire (SGRQ)).

Results We randomised 256 patients (128 telemonitoring): baseline characteristics were similar. Using an intention-to-treat analysis, there was no difference in time to admission between the groups (adjusted hazard ratio for admission (reference=tele-group) 1.03 (95\%CI 0.70 to 1.50). 61 patients in each group had an admission. There was no significant difference in the mean number of admissions/person (tele-group: 1.2 (SD 1.9), control: 1.1 (SD 1.6) $p=0.51$ ); bed days (tele-group: 9.4 (SD 19.1) vs control 8.8 (SD 15.9) $p=0.66$ ); or deaths (tele-group: 16, control 21. $\mathrm{p}=0.38$ ). Quality of life at 1 year was similar in both groups (SGRQ tele-group: 68.2 (16.3) vs usual: 67.3 (17.3), mean difference: 1.5 (95\% CI -1.5 to 4.5)).

Conclusion When both groups received optimised care, telemonitoring did not appear to reduce the time to a hospital admission, duration of hospital admissions or increase quality of life. The place of telemonitoring in clinical care may depend upon whether it offers efficiency savings by enabling professionals to monitor and support the care of more patients than using traditional means of communication.

ISRCTN number 96634935

Funding Chief Scientist Office of Scottish Government.

\section{S53 OUTCOMES OF THE REPNEU ENDOBRONCHIAL COILS FOR THE TREATMENT OF SEVERE EMPHYSEMA WITH HYPERINFLATION (RESET) TRIAL}

doi:10.1136/thoraxjnl-2012-202678.059

${ }^{1} Z$ Zoumot, ${ }^{1 S}$ Kemp, ${ }^{2} \mathrm{R}$ Chaudhuri, ${ }^{3} \mathrm{C}$ Caneja, ${ }^{2} \mathrm{~S}$ Bicknell, ${ }^{1} \mathrm{~N}$ Hopkinson, ${ }^{3} \mathrm{~S}$ Singh, ${ }^{2} \mathrm{E}$ Ross, 'P Shah. 'National Institute for Health Research Respiratory Biomedical Research Unit at the Royal Brompton and Harefield Hospital Trustlmperial College, London, United Kingdom; 'artnavel General Hospital, Glasgow, United Kingdom; ${ }^{3}$ Chelsea and Westminster Hospital NHS Foundation Trust, London, United Kingdom

Background The predominant pathophysiology in severe emphysema with gas trapping and hyperinflation is that of dynamic airway collapse on minimal expiratory effort. This limits the benefit from drug therapy. Safer and cheaper alternatives to lung volume reduction surgery (LVRS), which has success in selected patients with low exercise capacity and upper lobe-predominant emphysema, are being developed. Endobronchial valve treatment has been shown to be beneficial to patients with heterogenous disease in the absence of collateral ventilation. RePneu Lung Volume Reduction Coils (LVRCs) are self-actuating implantable devices composed of nitinol. They are implanted bronchoscopically using conscious sedation. The LVRC is delivered into targeted airways using fluoroscopic guidance, and when its sheath is removed recoils to it original pre-determined shape. Methods In a prospective randomised study of LVRCs on patients with severe emphysema and hyperinflation, 63 patients were screened at 3 centres in the United Kingdom with 23 randomised to treatment with LVRCs and 24 to best medical care (control). LVRC patients were initially treated in one lung, with the contralateral lung treated after one month if appropriate. The primary end point was the difference between treatment and control groups in the St. George's Respiratory Questionnaire (SGRQ) 90 days post-final treatment. The trial is registered with ClinicalTrials.gov (NCT01334307). 
Abstract S53 Table 1 Primary and Secondary Efficacy Outcomes in the Intent-to-Treat Population (Change from Baseline at 90 Days post Final Treatment)

\begin{tabular}{|c|c|c|c|c|}
\hline & RePneu Coil Treatment $(n=23)$ & Control $(n=24)$ & Between-Group Difference in Change from Baseline & P-value \\
\hline \multicolumn{5}{|c|}{ number ( $95 \%$ confidence interval) } \\
\hline \multicolumn{5}{|c|}{ Initial Analysist } \\
\hline \multicolumn{5}{|l|}{ Primary outcome } \\
\hline Mean change in SGRO & $-9.11(-14.59$ to -3.62$)$ & $1.43(-4.05$ to 6.92$)$ & $-10.54(-17.52$ to -3.56$)$ & 0.004 \\
\hline \multicolumn{5}{|l|}{ Secondary outcome } \\
\hline Mean change in TLC $(\mathrm{L})$ & $-0.36(-0.55$ to -0.18$)$ & $-0.25(-0.43$ to -0.07$)$ & $-0.11(-0.35$ to 0.12$)$ & 0.330 \\
\hline Mean change in RV (L) & -0.64 (0.92 to -0.37$)$ & $-0.29(-0.57$ to -0.02$)$ & $-0.35(-0.70$ to 0.00$)$ & 0.051 \\
\hline Mean change in 6-minute Walk Test (m) & 52.98 (29.18 to 76.78) & $-17.41(-41.21$ to 6.39$)$ & 70.39 (40.10 to 100.68$)$ & $<0.001^{\ddagger}$ \\
\hline Mean percent change in $\mathrm{FEV}_{1}$ & 14.85 (7.46 to 22.23$)$ & $2.04(-5.35$ to 9.42$)$ & 12.81 (3.41 to 22.21$)$ & $0.009^{\ddagger}$ \\
\hline
\end{tabular}

SGRQ denotes St. George's Respiratory Questionnaire, TLC total lung capacity, RV residual volume, and FEV, forced expiratory volume in 1 second.

${ }^{\dagger} P$-value determined by analysis of variance (ANOVA) with factors of treatment and site.

${ }^{\ddagger}$ Statistical significance via the Hochberg adjustment for multiplicity for secondary outcomes.

Results Significant improvements in the treatment group compared to control group were observed for the primary end point mean SGRQ $(\Delta-10.54$ points, $p=0.004)$, as well as secondary end points mean sixminute walk distance $(\Delta+70.39$ metres, $\mathrm{p}<0.001)$ and forced expiratory volume in one second $(\Delta+12.81 \%, p=0.009)$. Between group difference in change in mean residual volume did not reach significance $(\Delta-0.35$ litres, $p=0.051)$, despite a 0.64 litre reduction in the treatment group. There was a good safety profile with treatment.

Conclusions Treatment with endobronchial coils in patients with severe emphysema and hyperinflation significantly improves quality of life, exercise capacity and pulmonary function with a good safety profile. LVRCs present a novel, safe, and minimally invasive treatment option for patients with both homogenous and heterogenous emphysema, with benefits unaffected by collateral ventilation. A larger randomised controlled pivotal trial with longer follow-up is now needed. Funding shared by PneumRx and study sites.

\section{Regulating inflammation in acute lung injury}

\section{S54 THE ROLE OF PRE-RECEPTOR GLUCOCORTICOID METABOLISM IN REGULATING THE SEVERITY AND PERSISTENCE OF MURINE LUNG INJURY}

doi:10.1136/thoraxjnl-2012-202678.060

'S Lax, ${ }^{2} \mathrm{MR}$ Wilson, ${ }^{2} \mathrm{M}$ Takata, 'P Stewart, 'D Thickett. 'The University of Birmingham, Birmingham, UK; ${ }^{2}$ mperial College London, London, UK

Introduction Acute lung injury (ALI) is a major cause of respiratory failure in the critically ill patient. With a mortality rate of $40-60 \%$, $50 \%$ of survivors left with pulmonary impairment and no current licenced treatment there is a need for novel therapies. Our current research suggests that local steroid metabolism by alveolar macrophages is defective in ALI patients. As the predominant function of these cells is phagocytosis of apoptotic neutrophils during resolution of inflammation, we sort to investigate the effect of pre-receptor glucocorticoid metabolism in a murine model of ALI

Methods Using intra-tracheal instillations of LPS (50 $\mu \mathrm{g})$, we analysed the inflammatory response in wild type (WT) mice compared to those deficient in 11ß-hydroxysteroid dehydrogenase-1 (HSD-1 $\mathrm{KO})$. These mice specifically lack the enzyme which converts inactive cortisone to active cortisol. Cell infiltrates and expression of several inflammatory markers within bronchial lavage fluid (BALF), as well as tissue permeability and mouse oximetry were examined to evaluate the immune response and lung damage.

Results Intra-tracheal LPS challenge in WT mice induced a significant increase in lung permeability $(p=0.0153)$, infiltrating neutrophils $(p=0.0121)$ and recruitment of $\mathrm{CD} 11 \mathrm{c}^{+} \mathrm{CD} 11 \mathrm{~b}^{+}$monocytes $(p<0.0001)$, which was associated with significant hypoxia $(p<0.0001)$ compared to PBS-treated controls 48 hrs post-instillation.
The severity of acute neutrophilic inflammation and epithelial barrier defects as measured by lung permeability index were similar in WT and HSD-1 KO mice.

In contrast, during resolution of LPS-induced injury ( $72 \mathrm{hrs}$ postinstillation), HSD-1 KO mice had a significant accumulation of apoptotic neutrophils $(p=0.02)$ and a significant increase in $\mathrm{CD} 11 \mathrm{c}^{+} \mathrm{CD} 11 \mathrm{~b}^{+}$monocytes $(\mathrm{p}=0.0007)$ recruited into the lung compared to WT controls. Moreover, Luminex arrays revealed a significant increase in BALF levels of IL-1 $\beta(p=0.003)$ and dysregulation of IL-6, TNF $\alpha$ and CXCL1/KC during the time course.

Conclusion Our data indicate that insufficient alveolar glucocorticoid metabolism augments the duration but not initial severity of lung injury, possibly via a dysregulation of apoptotic neutrophil clearance and suggests that therapies targeting defective macrophage HSD-1 expression may have value in promoting the resolution of ALI

\section{S55 THE AMINOPEPTIDASE CD13 REGULATES HOMOTYPIC AGGREGATION OF NEUTROPHILS}

doi:10.1136/thoraxjnl-2012-202678.061

${ }^{1} \mathrm{CA}$ Fiddler, ${ }^{2} \mathrm{G}$ Murphy, ${ }^{1} \mathrm{AS}$ Cowburn, ${ }^{1} \mathrm{H}$ Parfrey, ER Chilvers'. 'Department of Medicine, University of Cambridge School of Clinical Medicine, Cambridge, UK; ${ }^{2}$ Department of Oncology, University of Cambridge, Cambridge, UK

Neutrophils are critical effector cells of the innate immune response and are recruited to sites of tissue injury in response to locally generated chemoattractants. Neutrophil recruitment is a highly regulated process involving complex interactions with the vascular endothelium and underlying tissue stroma. In addition to adhering to the endothelium, neutrophils can also self-associate, (a process known as homotypic aggregation - HA), which has been proposed to play a key role in disease states such as sepsis.

Aminopepetidase $\mathrm{N}$ or CD13 is a widely expressed membrane-bound metallopeptidase involved in the migration and invasion of cancer and endothelial cells. Neutrophils express CD13 on their cell surface, which is upregulated by TNF- $\alpha$, IL- 8 and fMLP We have shown that inhibition of aminopeptidase activity enhances the efficacy of TNF$\alpha$-induced neutrophil apoptosis (Cowburn et al. J Biol Chem 2006; 281:12458). Cross-linking anti-CD13 monoclonal antibodies (mAb) have been shown to induce HA of monocytic cells through PI3K activation (Mina-Osorio et al. J Leuk Biol 2006; 79:719). We hypothesised that CD13 may be involved in neutrophil migration and HA.

Using plasma-Percoll purified human neutrophils and a modified Boyden filtre assay we showed that IL-8 mediated neutrophil chemotaxis was not affected by either the CD13 mAb WM-15 or the aminopeptidase enzymatic inhibitor bestatin. In contrast, IL-8-mediated neutrophil migration through type 1 collagen gels was significantly impaired by WM-15 and MY7 mAbs, which both inhibit enzymatic activity and induce clustering of CD13. The non-clustering CD13 antibody WM-47 and bestatin had no effect. WM-15 and MY7 also 